Supporting Information for

\title{
MLCV: Bridging Machine Learning-Based Dimensionality Reduction and Free-Energy Calculation
}

Haochuan Chen, ${ }^{\dagger, \circ}$ Han Liu, ${ }^{\dagger, \circ}$ Heying Feng, ${ }^{\dagger, \circ}$ Haohao Fu, ${ }^{\dagger}$ Wensheng Cai, ${ }^{*},{ }^{\circ}$ and Xueguang Shao, ${ }^{* \dagger}$ Christophe Chipot, ${ }^{*}, \$, \$$

${ }^{\dagger}$ Research Center for Analytical Sciences, Frontiers Science Center for New Organic Matter, College of Chemistry, Nankai University, Tianjin Key Laboratory of Biosensing and Molecular Recognition, State Key Laboratory of Medicinal Chemical Biology, Tianjin 300071, China

Laboratoire International Associé CNRS and University of Illinois at Urbana-Champaign, UMR n7019, Université de Lorraine, BP 70239, F-54506 Vandœuvre-lès-Nancy, France

${ }^{\S}$ Department of Physics, University of Illinois at Urbana-Champaign, 1110 West Green Street, Urbana, Illinois 61801, United States

${ }^{\circ}$ Contribute equally 


\section{Compilation of MLCV}

The source code of the modified Colvars supporting dense neural networks is provided in colvars_neural_network.zip. The following instructions describe how to build the source code with NAMD:

1. Unzip colvars_neural_network.zip into an empty directory named colvars/;

2. Download the source code of NAMD 2.14 from its official website: https://www.ks.uiuc.edu/Development/Download/download.cgi?PackageName=N AMD, and then unzip the tarball. The source code of NAMD should be in NAMD_2.14_Source/;

3. Assuming that colvars/ and NAMD_2.14_Source/ are in the same parent directory, enter NAMD_2.14_Source/ and run “../colvars/updatecolvars-code.sh ./" to update the Colvars source code bundled with NAMD;

4. Compile NAMD as documented in the release note of NAMD (NAMD_2.14_Source/notes.txt), and run "make depends" before "make".

\section{Usage of MLCV}

To use MLCV in Colvars, users need to configure a NeuralNetwork component in the Colvars configuration file. The options of NeuralNetwork are listing below:

output_component: the index of the node in the last layer to be used as the final CV. This option accepts an integer starting from 0.

$>$ layeri_Weightsfile: the file containing the weights from layer $i$-1 to layer $i$. The letter $i$ should be replaced by the actual layer index starting from 1 . The weights are stored in a plain text file, where the number at $k$-th column and $l$-th row represents the weight from node $k$ at layer $i-1$ to the node $l$ at layer $i$.

$>$ layeri_Biasesfile: the file containing the biases of layer $i$. The letter $i$ should be replaced by the actual layer index starting from 1 . The biases are stored in a plain text file with only one column, where the number at $l$-th row represents 
the bias of node $l$ at layer $i$.

$>$ layeri_activation: the activation function for layer from layer $i-1$ to $i$. The letter $i$ should be replaced by the actual layer index starting from 1. Available choices for the activation function are tanh, linear and sigmoid.

$>$ layeri_custom_activation: a mathematical expression to define the activation function from layer $i-1$ to $i$. The letter $i$ should be replaced by the actual layer index starting from 1 . The input value must be written as $x$. For example, the ELU activation function can be expressed as select $(\operatorname{step}(x), \operatorname{alpha*}(\exp (x)-1), x)$. It should be noted that this option is mutually exclusive with the option layeri_activation above.

To use MLCV, one needs to provide beforehand the number of layers, the weights and the biases of each computational units at each layer, as well as the activation functions, and extract them into a plain-text file.

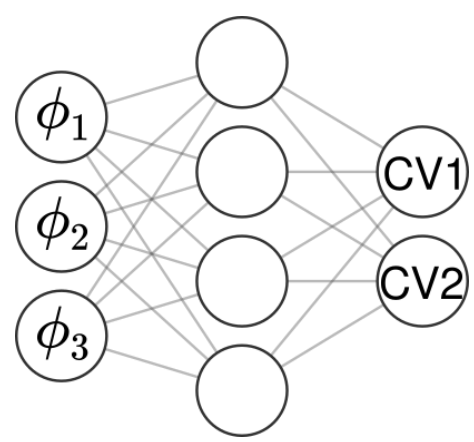

Figure S1. Schematic representation of the architecture of a three-layer dense neural network. $\phi_{1}, \phi_{2}$ and $\phi_{3}$ are some arbitrary CVs, which are already available in Colvars, for instance, the dihedral angles. CV1 and CV2 denote the CVs used in biased simulations.

For example, if one wants to use the architecture in Figure S1 of dense neural network as CVs, then the Colvars configuration file ought to look like that of Figure $\mathrm{S} 2$ : 

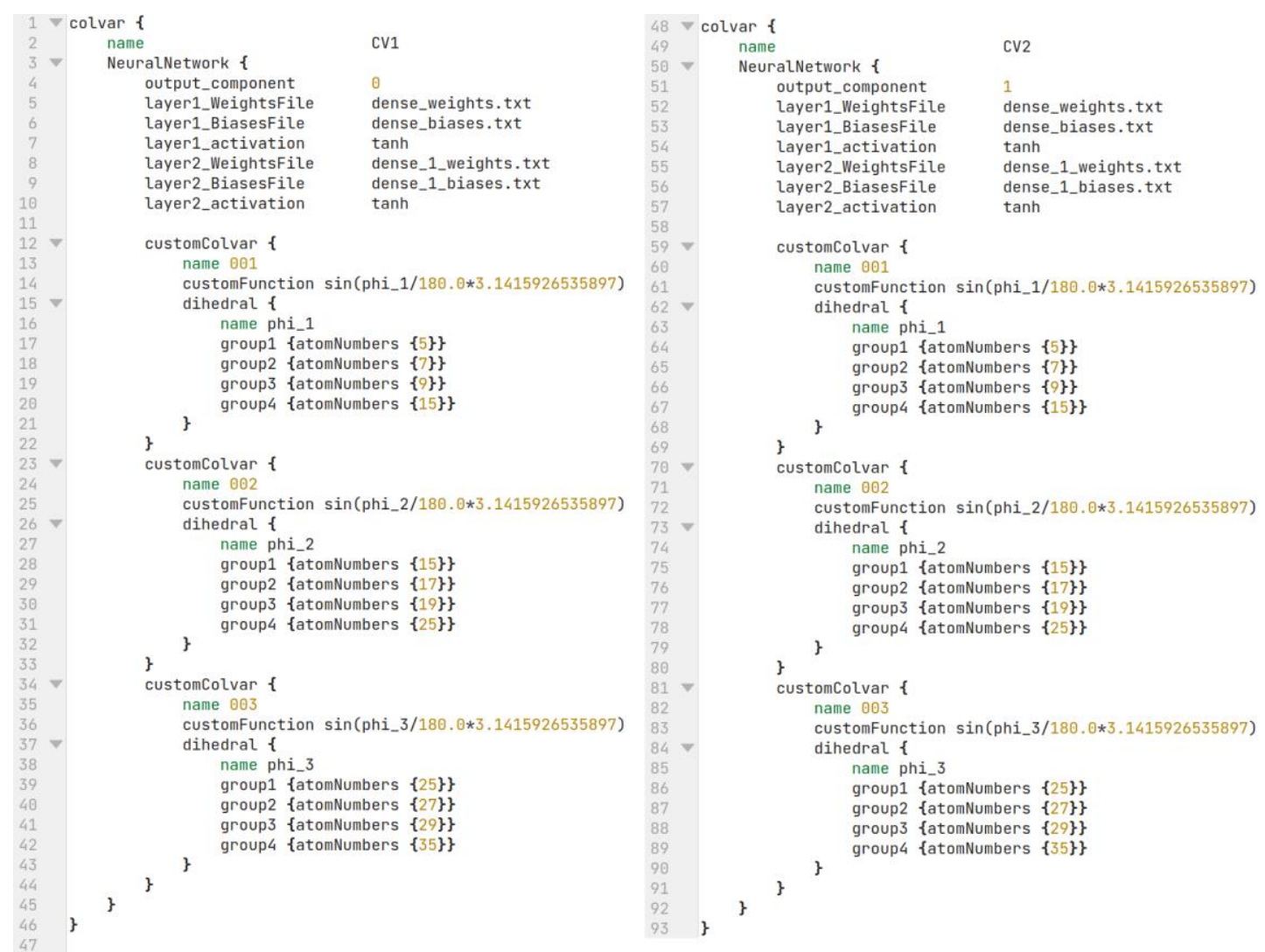

Figure S2. Example of a Colvars configuration file for the dense neural network schematized in Figure S1. The customColvar component are used to transform the dihedral angle CVs, which are already available in Colvars, into their corresponding sine values.

In Figure S2, a NeuralNetwork component corresponds to an output neuron shown in Figure S1. Since there are two output neurons used as CVs, two separate NeuralNetwork components with different indexes of output_component are defined. The weights of the three layers in a NeuralNetwork component are defined in the plain-text files named dense_weights.txt and dense_1_weights.txt. The file containing the weights has $m$ rows and $n$ columns, where $m$ and $n$ are, respectively, the number of the neurons of the next layer and that of the current layer. In the above instance, dense_weights.txt should consist of three columns separated by a space and four rows, and dense_1_weights.txt should consist of four columns separated by a space and two rows. By analogy, the file containing the biases possesses a similar structure, but consists only one column, and its number of rows matches the number of neurons of the next layer. In the aforementioned example, dense_biases.txt and dense_1_biases.txt consist, respectively, of four 
and two rows. The activation function of each layer is specified by either layeri_activation or layeri_custom_activation, where $i$ refers to the $i$-th layer. Although our example only uses a hyperbolic tangent (tanh), users can choose other activation functions, such as a linear function (linear) or a sigmoid function (sigmoid). Other activation functions can be defined via a custom mathematical function of variable $\mathrm{x}$. For example, to use the rectifier function-also known as rectified linear unit, or "ReLU"-at layer 1, the line "layer1_activation tanh" ought to be replaced by "layer1_custom_activation select (step (x), alpha* (exp (x)-1), $\mathrm{x})$ ". The trialanine example described in this work is provided in SI.zip (SI/trialanine/equil/wtmabf_ml/trialanine_ml.in).

\section{Simulation Speeds when Running NAMD with and without MLCV}

Table S1. Average simulation speed when running simulations with and without MLCV.

\begin{tabular}{lccccc}
\hline Test cases & Atoms & $\begin{array}{c}\text { Timestep } \\
\text { (fs) }\end{array}$ & $\begin{array}{c}\text { MLCV enabled } \\
\text { (ns/day) }\end{array}$ & $\begin{array}{c}\text { MLCV disabled } \\
\text { but } \\
\text { Colvars enabled } \\
\text { (ns/day) }\end{array}$ & $\begin{array}{c}\text { Colvars } \\
\text { disabled } \\
\text { (ns/day) }\end{array}$ \\
\hline Trialanine & 42 & 0.5 & $1112.1 \pm 122.6$ & $1236.2 \pm 69.3$ & $1190.0 \pm 49.5$ \\
Deca-alanine & 104 & 0.5 & $305.4 \pm 8.6$ & $312.5 \pm 5.3$ & $308.4 \pm 4.8$ \\
Chignolin & 8348 & 2 & $162.3 \pm 2.5$ & $171.7 \pm 3.2$ & $205.2 \pm 4.3$ \\
\hline
\end{tabular}

Table S1 compares the average speeds when running simulations with MLCV enabled, with MLCV disabled, albeit with Colvars enabled, as well as also without Colvars. As can be seen, simulations carried out with MLCV do not suffer from any marked performance loss. The trialanine benchmark was carried out on a laptop with a 16thread CPU (AMD Ryzen $75800 \mathrm{H}$ ), using the CPU version of NAMD. The decaalanine and chignolin benchmarks were carried out, respectively, on a server equipped with a 4-thread CPU (AMD EPYC 7551) and an NVIDIA GTX 1080 GPU, using the CUDA-accelerated NAMD. 


\section{Functional demonstration examples}

\section{Molecular Dynamics (MD) Simulations and Initial Training Data Generation.}

The structures of the three prototypical biomolecular models and the corresponding input CVs for the autoencoder (AE) model are shown in Scheme S1. The CVs used for trialanine, chignolin, and deca-alanine are the six dihedral angles, the sixteen dihedral angles between the peptide planes, and the six residue $i \rightarrow i+4$ intramolecular hydrogen-bond distances. To generate initial training trajectories, the trialanine was placed in vacuum, and 20-ns equilibrium and extended generalized adaptive biasing force (egABF) ${ }^{1}$ simulations were carried out with Amber ff14SB force field. ${ }^{2}$ For the chignolin, another 20-ns egABF simulation was performed with the Amber ff14SB force field in a $45 \times 45 \times 45 \AA^{3}$ TIP3P box $;^{3}$ for the deca-alanine, the same simulation time with the CHARMM22 protein force field in vacuum. ${ }^{4}$ In summary, the trajectories of the sine values of all backbone dihedral angles of the trialanine and chignolin, the residue $i \rightarrow i+4$ intramolecular hydrogen-bond distances of the deca-alanine were generated via these MD simulations, and were saved every 50 simulation steps.

All MD simulations were performed using parallel, scalable program NAMD 2.14 in this work. ${ }^{5}$ Visualization and analysis of MD trajectories were performed with program Visual Molecular Dynamics (VMD). ${ }^{6}$ In the egABF simulations, the coordinates were defined as dihedral angle between the peptide planes (ranging from $180^{\circ}$ to $180^{\circ}$ ) for the trialanine and chignolin, and residue $i \rightarrow i+4$ intramolecular hydrogen-bond distances (ranging from 2 to 20) for the deca-alanine, respectively. Langevin dynamics and the Langevin piston method maintained the temperature and pressure at $300 \mathrm{~K}$ and 1 atm, respectively. ${ }^{7,8}$ The SETTLE $^{9}$ and the SHAKE/RATTLE ${ }^{10,11}$ algorithms were used to constrain in water and other molecule respectively, covalent bonds involving hydrogen atoms to their equilibrium length. A $12-\AA$ cutoff was used to truncate Lennard-Jones and short-range Coulombic interactions using the CHARMM22 protein force field, and 9- $\AA$ cutoff for the Amber ff14SB force field. For short- and long-range interactions of explicit-solvent simulations, the equations of motion were integrated with a time step of 2 and 4 fs using 
the r-RESPA multiple-time-step algorithm. ${ }^{12}$

Training of Autoencoder. In initial training trajectories of the three test cases, we filtered out the simulation frames where the free energies are less than 3,13 and 10 $\mathrm{kcal} / \mathrm{mol}$ from the egABF simulations as the initial training data for trialanine, decaalanine and chignolin, respectively; in the case of trialanine, the initial training data are sub-sampled from the equilibrium simulation to make sure that its size matches the size from the egABF simulation. The initial training data were randomly divided into training, verification and test sets (see Table S3). The training set was firstly used as the input data of neural network to train the AE. Optimized neural network workflow of the $\mathrm{AE}$ for training the trialanine as an example is shown in Figure S3. The corresponding numbers of neurons are listed in Table S2. As shown in Figure S4, S6 and S8, the losses of the training and the verification sets do not decrease after 10,000, 13,000 and 13,000 epochs, indicating that training of the AE model have converged. In this work, the training sessions (epoch), and the batch_size were set to 50,000 and 256, respectively. Early stopping strategy, that is, terminating training when training epochs either reach the maximum number or the mean absolute error (MAE) evaluated over the validation set no longer decreases for 10 continuous epochs, was employed to prevent overfitting. ${ }^{13}$ At the end of the training, the accuracy of the final reaction coordinate model was determined using a test set.

Table S2. Numbers of neurons of the autoencoders.

\begin{tabular}{ccccccc}
\hline \multirow{2}{*}{ Layer } & \multicolumn{2}{c}{ Trialanine } & \multicolumn{2}{c}{ Deca-alanine } & \multicolumn{2}{c}{ Chignolin } \\
\cline { 2 - 7 } & Neurons & $\begin{array}{c}\text { Activation } \\
\text { function }\end{array}$ & Neurons & $\begin{array}{c}\text { Activation } \\
\text { function }\end{array}$ & Neurons & $\begin{array}{c}\text { Activation } \\
\text { function }\end{array}$ \\
\hline 1 & 6 & & 6 & & 16 & \\
2 & 12 & Tanh & 12 & Tanh & 4 & Tanh \\
3 & 1 & Tanh & 1 & Tanh & 2 & Sigmoid \\
4 & 6 & Tanh & 6 & Tanh & 4 & Tanh \\
5 & 12 & Tanh & 12 & Tanh & 16 & Tanh \\
\hline
\end{tabular}


Table S3. Numbers of frames of training, verification and test sets for the three test cases.

\begin{tabular}{lccc}
\hline & Trialanine & Deca-alanine & Chignolin \\
\hline Training set & 18,000 & 36,000 & 7,200 \\
Verification set & 4,500 & 9,000 & 1,800 \\
Test set & 2,500 & 5,000 & 1,000 \\
Total & 25,000 & 50,000 & 10,000 \\
\hline
\end{tabular}

Free-Energy calculations. All free-energy calculations along the learned CVs from the AE were carried out with the well-tempered meta-eABF (WTM-eABF) algorithm ${ }^{14}$ using MLCV. The simulation time amounted to $10 \times 100 \mathrm{~ns}$ and $900 \mathrm{~ns}$ for deca-alanine and chignolin, respectively. Since the value of the activation function is between -1 and 1 or 0 and 1 , the transition pathway extended from -1 to 1 or 0 and 1 . For the decaalanine, a 20- $\mu$ s multiple-walker ABF simulation was carried out, using the WTMeABF algorithm along the end-to-end distance chosen as the reaction coordinate. The timestep was set to $0.5 \mathrm{fs}$ in vacuum, and $2 \mathrm{fs}$ in the aqueous solution.

A

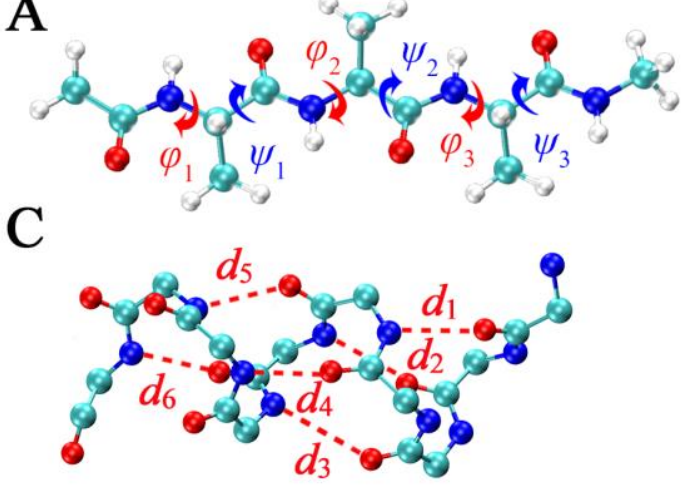

B

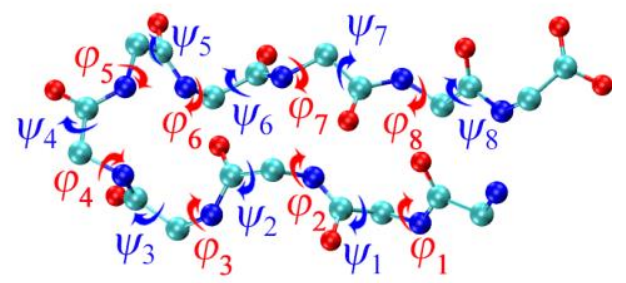

Scheme S1. Structures and dihedral angles between peptide planes of (A) trialanine, (B) chignolin, and (C) distances of residue $i \rightarrow i+4$ intramolecular hydrogen bonds of deca-alanine. 


\section{Isomerization of Trialanine in Vacuum}

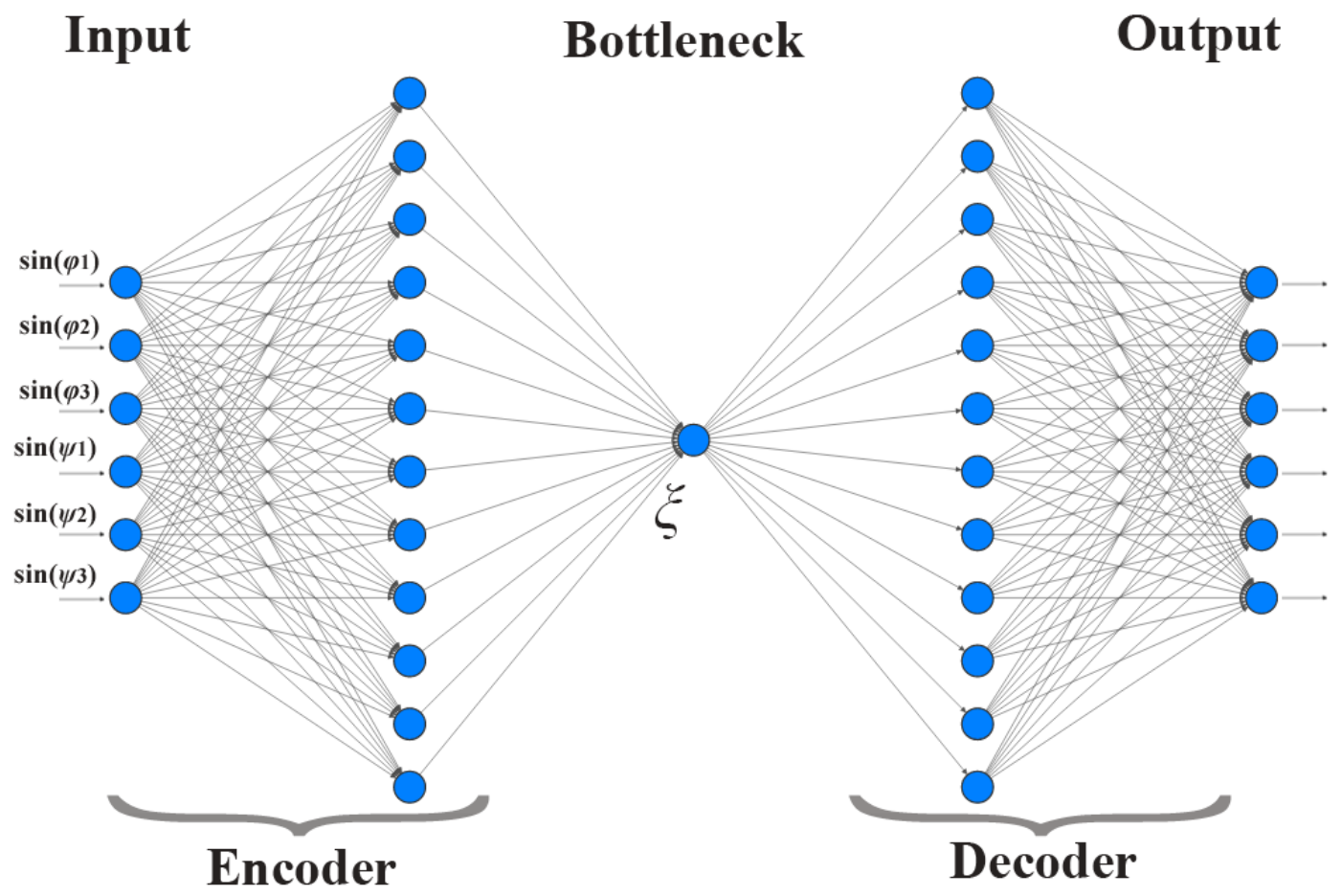

Figure S3. Neural network workflow of the AE for training the trialanine.

\section{Implementation steps and commands:}

\section{(1) Generating Initial Training Data}

Equilibrium and egABF simulations of the trialanine were carried out to generate the trajectories of the sine values of all backbone dihedral angles (.colvars.traj file) using program NAMD 2.14. ${ }^{5}$ Then the data of the sine values of backbone dihedral angles in low-energy regions were chosen as initial training data. The input files are provided in SI/trialanine/equil/traj_to_learn and SI/trialanine legABF/traj_to_learn folders of SI.zip.

\section{(2) Training of $\mathrm{AE}$}

Input and output files for training of the $\mathrm{AE}$ are provided in SI/trialanine/equil/learn and SI/trialanine/egABF/learn. Users can run following command to test:

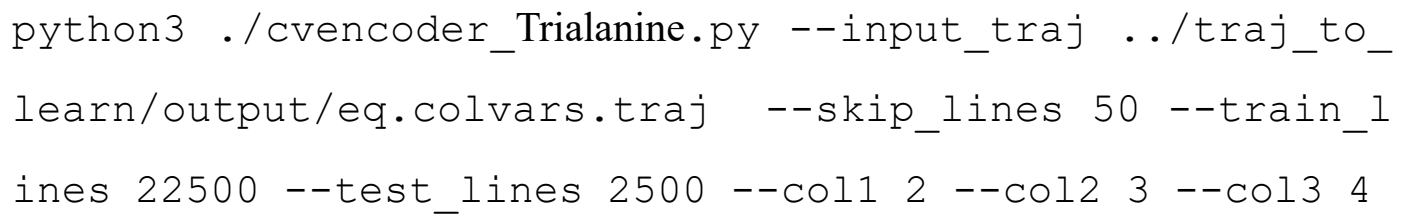


$--\operatorname{col} 4 \quad 5--\operatorname{col} 5 \quad 6--\operatorname{col} 67$

After the training, the weights and bias of the learned AE are extracted using the show_weights.py script, and is written to a TXT file (dense_*_biases.txt and dense_*_weights.txt).

\section{(3) Free-energy calculations based on the learned CVs}

Input and output files for free-energy calculations are provided in SI/trialanine/equil/wtmabf_ml and SI/trialanine/egABF/wtmabf_ml. The freeenergy calculation describing the conformational change of the trialanine was performed using NAMD $2.14^{5}$ including the updated Colvars module. When carrying out the free-energy calculations, the weights, bias and activation functions of the AE need to be provided to the Colvars configuration file (trialanine_ml.in).

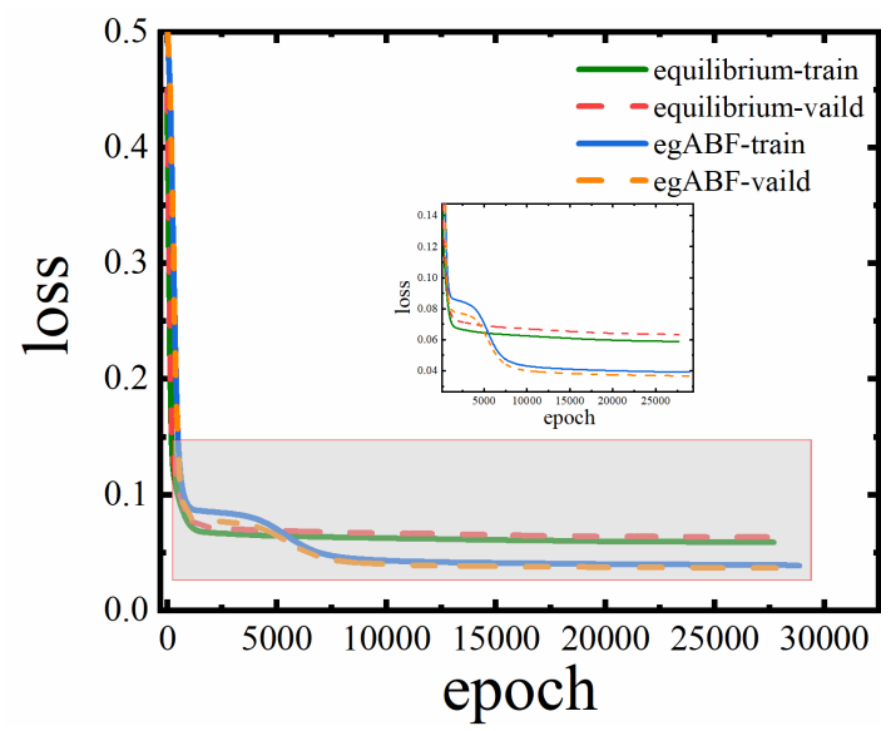

Figure S4. The epoch evolution of the loss of training and verification sets for the trialanine. 

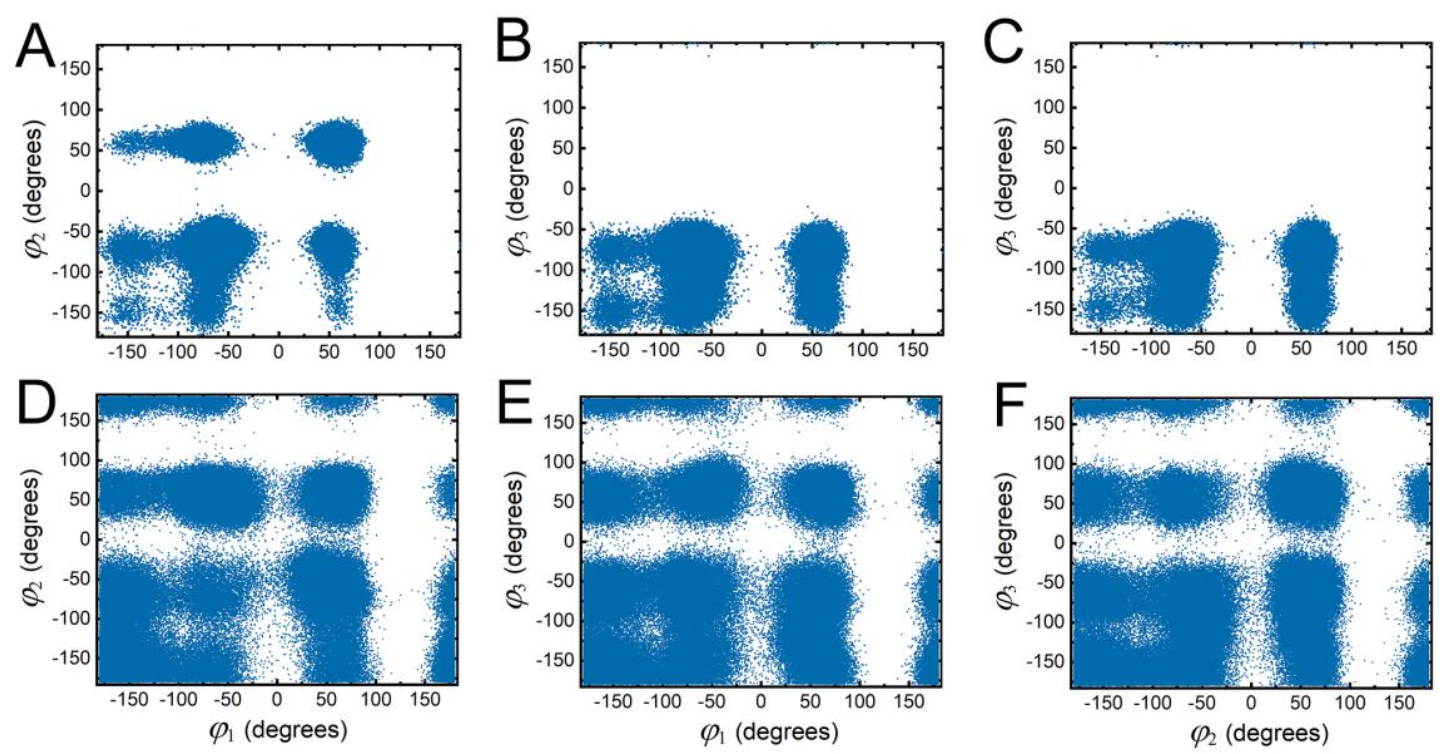

Figure S5. Sampling of the initial trajectories obtained from 20-ns (A-C) equilibrium, and (D-F) egABF simulations of trialanine.

\section{Folding of Chignolin in the Aqueous Solution}

\section{Implementation steps and commands:}

Steps (1)-(3) are the same as the trialanine, except that we use the sine values of sixteen dihedral angles between the peptide planes of the chignolin as the input CVs for training.

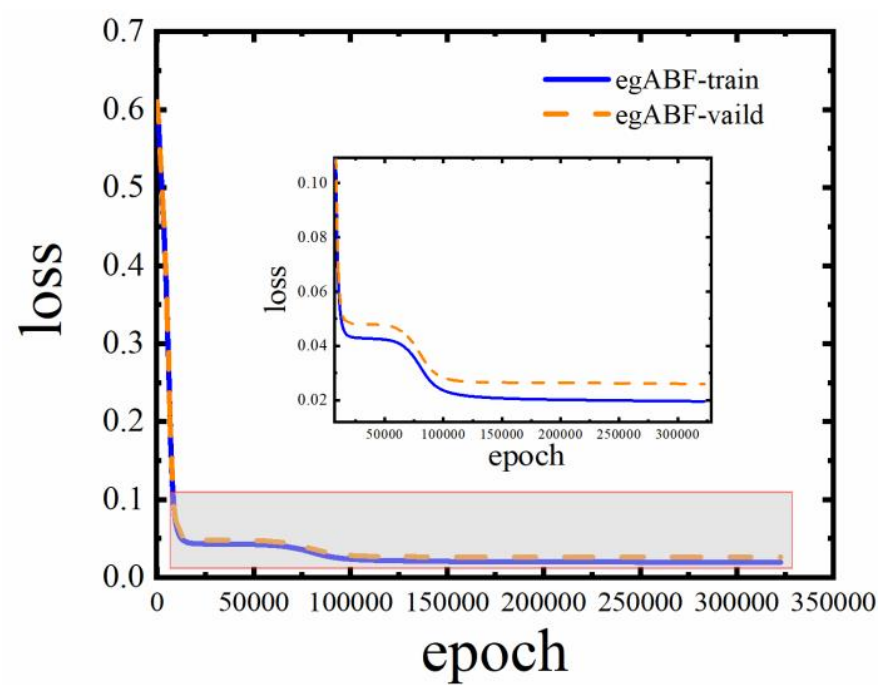

Figure S6. The epoch evolution of the loss of training and verification sets for the chignolin.

Training of the Autoencoder: In order to investigate the impact of the architecture of the $\mathrm{AE}$ on the learned $\mathrm{CV}$, we have trained another $\mathrm{AE}$ model with 32 computational 
units in the second layer. After training the AE, the PMF was then computed along the learned $\mathrm{CV}$, and then reweighted along the same hydrogen-bond distances, $d_{1}$ and $d_{2}$, as reported in Figure 3B. As can be seen in Figure S7, although the reweighted freeenergy landscape also features three local minima, the positions of these minima on the landscape do not quite match those reported by Oshima et al. ${ }^{15}$ In contrast, the locations of the three minima in Figure 3A, resulting from the free-energy calculation with an $\mathrm{AE}$ model built using four units in the second layer, are in close agreement with the study of Oshima et al. ${ }^{15}$ In summary, using an AE with an architecture of 16-4-2-4-16 allows the conformational equilibrium of chignolin to be described satisfactorily.

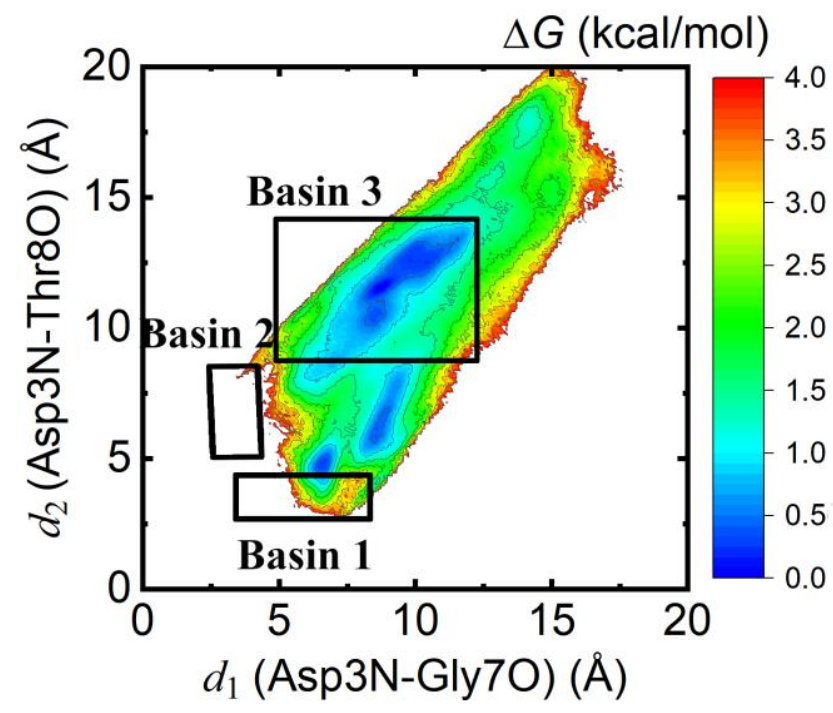

Figure S7. Free-energy landscape of chignolin along $d_{1}$ and $d_{2}$, reweighted from the free-energy calculation using CVs learned from an AE of an architecture of 16-32-232-16.

\section{Folding of Deca-alanine in Vacuum}

\section{Implementation steps and commands :}

Steps (1)-(3) are the same as above, except that we use the six $i \rightarrow i+4$ intramolecular hydrogen-bond distances of the deca-alanine as the input CVs for training. 


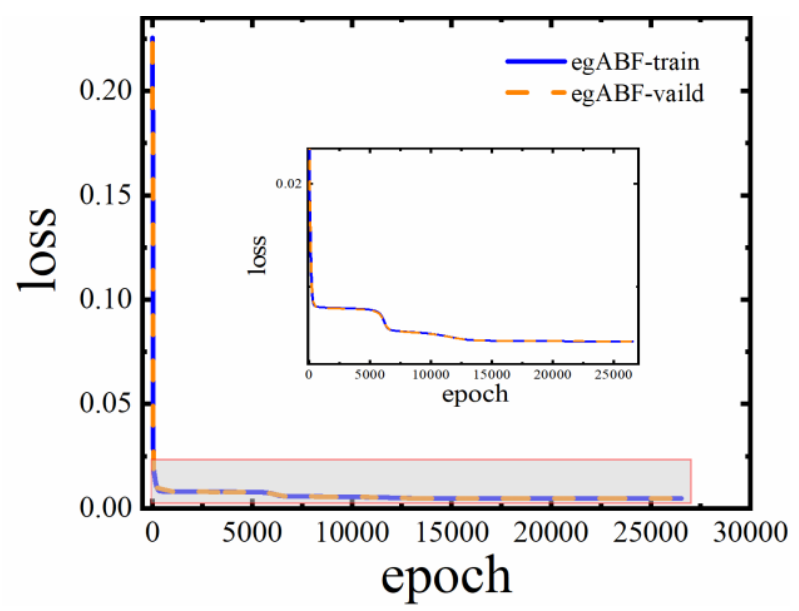

Figure S8. The epoch evolution of the loss of training and verification sets for the decaalanine.

In the case of deca-alanine, the six CVs describing the conformational transitions, namely the intramolecular hydrogen bonds formed between residue $i$ and residue $i+4$ $(i \rightarrow i+4)$, were reduced to a one-dimensional CV by the AE. After ten independent 100-ns WTM-eABF simulations along the learned CV, the free-energy profiles were then reweighted to the end-to-end distance, $\xi$, often used in previous studies of the reversible folding of deca-alanine, ${ }^{16}$ and their average is shown as the red curve in Figure S9, together with the reference PMF determined from a $20-\mu$ s multiple-walker ABF simulation. ${ }^{17}$ The comparison in Figure S9 reveals that although the reweighted PMF from the free-energy calculation using the learned CV only features three metastable states, it still strikingly resembles the reference free-energy profile, especially at $\xi$ ranging between 10 and $18 \AA$. The deviation of the PMF using the learned $\mathrm{CV}$ from the reference free-energy profile, which is somewhat more apparent for $\xi$ ranging between 4 and $10 \AA$, may be ascribed to insufficient training of the data within the timescale of the egABF simulation. It should be noted that $\xi$ in the range of 4-10 $\AA$ is a particularly degenerate variable, with very high conformational entropy, i.e., one value of the $\mathrm{CV}$ corresponds to many distinct conformations. Generation of the PMF in this region, using the end-to-end distance of the peptide chain, has always proven to be a numerical challenge. ${ }^{18}$ The present case study, nonetheless, suggests that hydrogenbond distances also constitute a relevant CV input for MLCV applications. 


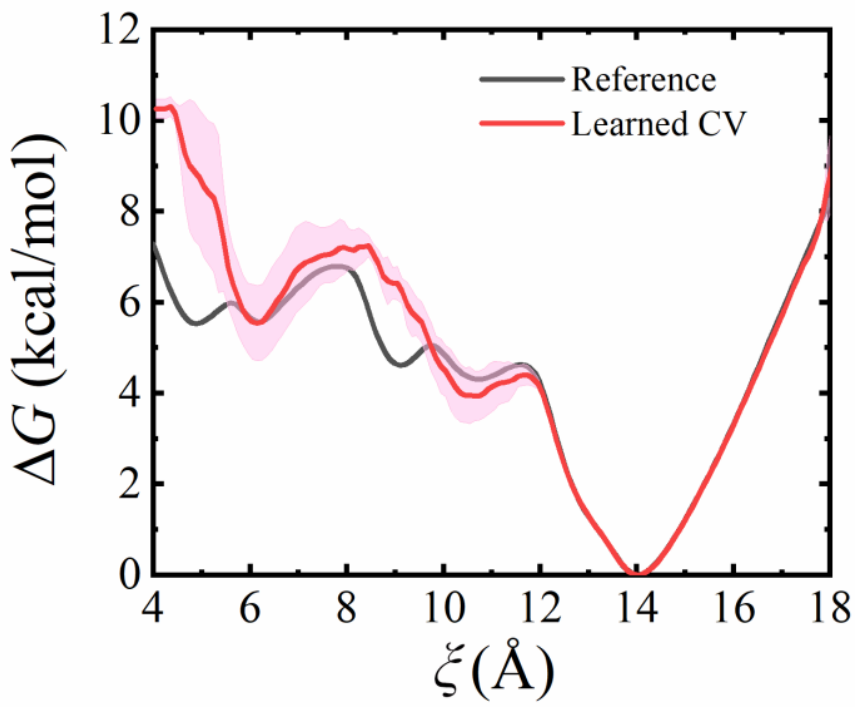

Figure S9. Free-energy profile along the end-to-end distance, $\xi$, of deca-alanine determined from a $20-\mu$ s multiple-walker ABF simulation (black line), and the average free-energy profile obtained from ten 100-ns WTM-eABF simulations based on the learned CVs (red line). The error bars inferred from the ten simulations are shown as a shaded area around the curves.

\section{REFERENCES}

(1) Zhao, T.; Fu, H.; Lelièvre, T.; Shao, X.; Chipot, C.; Cai, W. The Extended Generalized Adaptive Biasing Force Algorithm for Multidimensional Free-Energy Calculations. J. Chem. Theory Comput. 2017, 13, 1566-1576.

(2) Maier, J. A.; Martinez, C.; Kasavajhala, K.; Wickstrom, L.; Hauser K. E.; Simmerling, C. ff14SB: Improving the Accuracy of Protein Side Chain and Backbone Parameters from ff99SB. J. Chem. Theory Comput. 2015, 11, 3696-3713.

(3) Jorgensen, W. L.; Chandrasekhar, J.; Madura, J. D.; Impey, R. W.; Klein, M. L. Comparison of Simple Potential Functions for Simulating Liquid Water. J. Chem. Phys. 1983, 79, 926-935.

(4) MacKerell Jr, A. D.; Bashford, D.; Bellott, M.; Dunbrack, R. L.; Evanseck, J. D.; Field, M. J.; Fischer, S.; Gao, J.; Guo, H.; Ha, S.; Joseph-McCarthy, D.; Kuchnir, L.; Kuczera, K.; Lau, F. T. K.; Mattos, C.; Michnick, S.; Ngo, T.; Nguyen, D. T.; Prodhom, B.; Reiher, W. E.; Roux, B.; Schlenkrich, M.; Smith, J. C.; Stote, R.; Straub, J.; Watanabe, M.; Wiórkiewicz-Kuczera, J.; Yin, D.; Karplus, M. All-Atom Empirical Potential for Molecular Modeling and Dynamics Studies of Proteins $\dagger$. J. Phys. Chem. B 1998, 102, 3586-3616.

(5) Phillips, J. C.; Hardy, D. J.; Maia, J. D. C.; Stone, J. E.; Ribeiro, J. V; Bernardi, R. C.; Buch, R.; Fiorin, G.; Hénin, J.; Jiang, W.; McGreevy, R.; Melo, M. C. R; Radak, 
B. K.; Skeel, R. D.; Singharoy, A.; Wang, Y.; Roux, B.; Aksimentiev, A.; LutheySchulten, Z.; Kalé, L. V.; Schulten, K.; Chipot, C.; Tajkhorshid, E. Scalable Molecular Dynamics on CPU and GPU Architectures with NAMD. J. Chem. Phys. 2020, 153, 44130 .

(6) Humphrey, W.; Dalke, A.; Schulten, K. VMD: Visual Molecular Dynamics. J. Mol. Graphics 1996, 14, 33-38.

(7) Uhlenbeck, G. E.; Ornstein, L. S. On the Theory of the Brownian Motion. Phys. Rev. 1930, 36, 823-841.

(8) Feller, S. E.; Zhang, Y.; Pastor, R. W.; Brooks, B. R. Constant Pressure Molecular Dynamics Simulation: The Langevin Piston Method. J. Chem. Phys. 1995, 103, 4613-4621.

(9) Miyamoto, S.; Kollman, P. A. SETTLE: An Analytical Version of the SHAKE and RATTLE Algorithm for Rigid Water Models. J. Comput. Chem. 1992, 13, 952-962.

(10) Ryckaert, J. P.; Ciccotti, G.; Berendsen, H. J. C. Numerical Integration of the Cartesian Equations of Motion of a System with Constraints: Molecular Dynamics of n-Alkanes. J. Comput. Phys. 1977, 23, 327-341.

(11) Andersen, H. C. Rattle: A "Velocity" Version of the Shake Algorithm for Molecular Dynamics Calculations. J. Comput. Phys. 1983, 52, 24-34.

(12) Tuckerman, M.; Berne, B. J.; Martyna, G. J. Reversible Multiple Time Scale Molecular Dynamics. J. Chem. Phys. 1992, 97, 1990-2001.

(13) Yuan, Y.; Rosasco, L.; Caponnetto, A.; On early stopping in gradient descent learning. Constr. Approx. 2007, 26, 289-315.

(14) Fu, H.; Shao, X.; Cai, W.; Chipot, C. Taming Rugged Free Energy Landscapes Using an Average Force. Acc. Chem. Res. 2019, 52, 3254-3264.

(15) Oshima, H.; Re, S.; Sugita, Y. Replica-Exchange Umbrella Sampling Combined with Gaussian Accelerated Molecular Dynamics for Free-Energy Calculation of Biomolecules. J. Chem. Theory Comput. 2019, 15, 5199-5208.

(16) Hénin, J.; Chipot, C. Overcoming Free Energy Barriers Using Unconstrained Molecular Dynamics Simulations. J. Chem. Phys. 2004, 121, 2904-2914.

(17) Minoukadeh, K.; Chipot, C.; Lelièvre, T. Potential of Mean Force Calculations: A Multiple-Walker Adaptive Biasing Force Approach. J. Chem. Theory Comput. 2010, 6, 1008-1017.

(18) Comer, J.; Roux, B.; Chipot, C. Achieving Ergodic Sampling using ReplicaExchange Free-Energy Calculations. Mol. Simul. 2014, 40, 218-228. 